\title{
COMORBILIDAD DEL PACIENTE HOSPITALIZADO EN EL SERVICIO DE GERIATRÍA EN LA FUNDACIÓN HOSPITAL SAN CARLOS
}

\section{COMORBIDITY OF THE PATIENT HOSPITALIZED IN GERIATRIC'S SERVICE AT SAN CARLOS HOSPITAL FOUNDATION}

\author{
Paola Jimena Contreras-Acevedo ${ }^{1}$, Luz Dalila Vargas ${ }^{2}$, Danny Wilson Sanjuanelo Corredor ${ }^{3}$, Juan Pablo Robayo ${ }^{4}$, Carmen \\ Lucía Niño Cardozo ${ }^{5}$
}

\begin{abstract}
${ }^{1}$ Estudiante de Enfermería. Universidad Ciencias Aplicadas y Ambientales U.D.C.A, e-mail: pcontreras@udca.edu.co; ${ }^{2}$ Enfermera M.Sc en Enfermería, Profesora Programa de Enfermería. Universidad Ciencias Aplicadas y Ambientales U.D.C.A, e-mail: luvargas@udca.edu.co; ${ }^{3}$ Ingeniero Agrónomo, Facultad de Ciencias. Universidad Ciencias Aplicadas y Ambientales U.D.C.A, e-mail: dsanjuanelo@udca.edu.co; ${ }^{4}$ Médico, Magíster en Calidad y Gestión Integral, Director de Calidad y Seguridad del Paciente. Fundación Hospital San Carlos, e-mail: juanprobayop@gmail.com; ${ }^{5}$ Enfermera M.Sc en Bioética, Profesora Programa de Enfermería. Universidad Ciencias Aplicadas y Ambientales U.D.C.A, e-mail: cnino@udca.edu.co
\end{abstract}

Rev. U.D.C.A Act. \& Div. Cient. 19(2): 267-273, Julio-Diciembre, 2016

\section{RESUMEN}

La comorbilidad es la existencia de una entidad clínica adicional a una enfermedad bajo estudio, que ocurre durante la hospitalización de un paciente. Genera prolongación en la estancia hospitalaria, impactando sobre la terapéutica, el pronóstico de la enfermedad a mediano y largo plazo y la demanda del servicio en salud. Se realizó un estudio cuantitativo de tipo analítico, retrospectivo. Se revisaron 93 historias clínicas y se aplicó un análisis de varianza, utilizando el lenguaje de programación $\mathrm{R}$, para determinar la prevalencia de diagnósticos de comorbilidad en la población evaluada. Se identificaron 151 diagnósticos de egresos. Las más representativas fueron: Hipertensión Arterial: 10,4\%; Enfermedad Pulmonar Obstructiva Crónica: 6,1\%; Diabetes Mellitus Tipo II: 4,1\%; Infección de Vías Urinarias: 3,6\%. El análisis de la prevalencia mostró la presencia de 32 diagnósticos que prolongaron la estancia hospitalaria, principalmente: Enfermedad Renal Crónica, Neumonía Asociada al Cuidado de la Salud, Fístula Recto Vaginal, Hipoalbuminemia y Obstrucción Urinaria. La comorbilidad encontrada en pacientes geriátricos hospitalizados en la Fundación Hospital San Carlos corresponde a enfermedades agudas y crónicas de aparición repentina durante la estancia hospitalaria. Su vínculo con los estados de salud iniciales se debe aún establecer.

Palabras clave: Anciano, comorbilidad, enfermedad crónica, hospitalización.

\section{SUMMARY}

Comorbidity is the existence of a clinical entity in addition to a disease under study. It occurs during a patient hospitalization and generates longer hospitalization time, impacts therapeutic, medium- and long-term disease prognosis as well as the health service demand. A quantitative study of analytical, retrospective was carried out. Ninety-three medical records were reviewed and a variance analysis, using the programming language $R$, to determine the prevalence of comorbid diagnoses in the study population was performed. 151 discharge diagnoses were determined, being the most representative: Hypertension $10.4 \%$, Chronic Obstructive Pulmonary Disease 6.1\%, Type II Diabetes Mellitus 4.1\%, and Urinary Tract Infection 3.6\%. The prevalence analysis showed the presence of 32 diagnoses that led to the prolongation of hospital stay. Most importantly: Chronic Kidney Disease, Pneumonia Associated with Health Care, Recto Vaginal Fistula, Hypoalbuminemia, and Urinary obstruction. The data found indicated that comorbidity found in geriatric patients hospitalized in the Hospital San Carlos Foundation, correspond to sudden onset of chronic and acute illness during the hospital staying. Whether they are linked to the initial health status, is to be determined.

Key words: Aged, comorbidity, chronic disease, day care, medical. 


\section{INTRODUCCIÓN}

El término comorbilidad fue introducido a la medicina por Alvan Feinstein, en 1970, como la existencia de una entidad clínica adicional distinta, que ocurre durante el curso hospitalario de un paciente con una enfermedad bajo estudio (Rosas et al. 2011). Karlamangla et al. (2007), la definen como el impacto total de la disfunción biológica e, incluyen, no sólo las enfermedades evidentes, sino también procesos subclínicos, que no llegan al diagnóstico de enfermedad por los criterios actuales. Martínez \& Gaminide (2011) destacan, además de definir en estos términos la comorbilidad, la importancia de la apropiada cuantificación de la ocurrencia en el paciente anciano.

Para efectos de otros estudios, se define como la presencia de diferentes enfermedades que acompañan, a modo de satélite, a una enfermedad protagonista crónica (Bernabeu et al. 2014); sin embargo, el término ha evolucionado y, en la actualidad, se evidencian dos tipos de comorbilidad: la clásica, definida por Feinstein (1970) y la moderna o multimorbilidad, introducida a la literatura científica en los años noventa, como cualquier combinación de una enfermedad crónica con al menos otra enfermedad aguda o crónica, o con un factor psicosocial asociado o no o con un factor somático (Fernández \& Bustos, 2016).

Posteriormente, surgen modelos conceptuales de comorbilidad moderna o multimorbilidad, propuestos por Valderas et al. (2009) entre otros. Estos modelos tienen la particularidad de ser multicausales e incorporan diversas interrelaciones entre los factores etiológicos, incluidos los determinantes sociales de los padecimientos involucrados en la multimorbilidad. Esta definición, permite tener un punto de referencia conceptual muy distinto, de particular importancia en los adultos mayores (Fernández \& Bustos, 2016).

La presencia de más de una afección de salud es muy frecuente en la población anciana, entre el 55 y $98 \%$ en personas de más de 60 años, según las series (Laporte, 2012); se incrementa con la edad y ocurre más en las mujeres y en personas con bajos ingresos. Se pueden producir infinidad de combinaciones y se ha intentado realizar una aproximación sobre modelos etiológicos de comorbilidad, analizando la sincronía de las mismas y la interacción etiológica (Laporte, 2012).

En el ámbito asistencial, hace años se ha definido a un grupo de pacientes con alta multimorbilidad, denominándolos pacientes pluripatológicos, en el entorno español o afectos de múltiples condiciones crónicas, en el entorno anglosajón, definiendo, incluso, unos criterios diagnósticos (Boult \& Wieland, 2010). La pluripatología hace referencia a aquellos pacientes que presentan dos o más enfermedades y también una especial susceptibilidad y fragilidad clínica. Serviría para identificar a pacientes que, como consecuencia de su enfermedad, presentan un riesgo elevado de caer en la cascada de la dependencia y la discapacidad y aquellos que, aun sin dependencia franca, presentan diferentes enfermedades crónicas, con síntomas continuos y agudizaciones frecuentes (Alonso et al. 2013).

Las Enfermedades Crónicas no Trasmisibles (ECNT), se definen como enfermedades con un proceso de evolución prolongada, que no se resuelven espontáneamente y rara vez alcanzan una cura completa. Tiene una etiología múltiple y un desarrollo poco predecible y presentan múltiples factores de riesgo (Robledo \& Escobar, 2010). Las ECNT, se pueden presentar a lo largo de la vida, con mayor incidencia en las personas mayores de 65 años, quienes son el grupo etario de mayor crecimiento en los últimos años y, actualmente, representan el 1,3\% de la población mundial; en el 2050, se prevé que serán el 4,4\%, más de 370 millones de personas (Gómez et al. 2014).

La población mundial está envejeciendo a pasos acelerados. Según la OMS, entre 2000 y 2050, la proporción de los habitantes del planeta mayores de 60 años se duplicará, pasando del 11 al 22\%. En América, se estima que para el 2020 habrá 200 millones de personas de más de 60 años y esta cifra subirá a 310 millones, en 2050 (OMS, 2016).

El proceso de envejecimiento está constituido por diferentes cambios que son complejos. En el plano biológico, el envejecimiento está asociado con la acumulación de una gran variedad de daños moleculares y celulares (OMS, 2015); a su vez, sobreviniendo importantes cambios morfológicos y funcionales en los sistemas cardiovascular, renal, nervioso central, muscular y metabolismo de la glucosa, asociados a la edad, aumenta el riesgo de enfermedades crónicas (Salech et al. 2012) y sus consecuencias. Por tanto, se reconoce que existen otras características que definen a la morbilidad geriátrica, como la comorbilidad ( 2 o más enfermedades concomitantes) (Arango et al. 2012), que hace imperativa la identificación de ésta en la población, por parte del personal sanitario, permitiendo garantizar la optimización y la calidad de la atención en salud.

Cada año, 30 millones de personas en el mundo mueren por causa de las ECNT. Para el 2012, causaron 38 millones (68\%), de los 56 millones de defunciones registradas en el año. Más del $40 \%$ de ellas (16 millones) fueron muertes prematuras, ocurridas antes de los 70 años de edad.

Las ECNT son comparadas con una epidemia mundial, dada la morbimortalidad que revela un impacto creciente y desproporcionado, que incide en el desarrollo de los pueblos y genera una mayor demanda de servicios de salud de alta 
complejidad y de calidad, para la atención de la población. Es así, como más del $80 \%$ de las muertes son causadas por enfermedades cardiovasculares y diabetes y alrededor del $90 \%$, por enfermedades pulmonares obstructivas (OMS, 2014); en Colombia, más de 110 mil personas fallecen por enfermedades crónicas (PAHO, 2015). Según datos estadísticos, las 5 primeras causas de morbilidad en la población mayor de 65 años y más son: enfermedades isquémicas del corazón $(21,1 \%)$, enfermedades crónicas de las vías respiratorias inferiores $(9,3 \%)$, enfermedades cerebrovasculares $(8,2 \%)$, enfermedad hipertensiva $(4,5 \%)$ y diabetes mellitus $(5,2 \%)$ (MinSalud y Protección Social, 2012).

Durante el 2011, en Colombia, se registraron 80.048 .565 atenciones; el 93,6\% (74.925.456) fueron consultas; el 4,5\% (3.602.185), urgencias y el 1,2\% (960.582), hospitalizaciones. Asimismo, en la región de Bogotá-Cundinamarca, se atendieron un total de 19.212.629 personas, donde el 84,7\% (16.273.096) fueron consultas; el 10,1\% (1.940.475), urgencias y el 4,8\% (9.22.206), hospitalizaciones; además, produjo el $22 \%$ de la mortalidad del país durante el 2010. La principal causa de muerte estuvo relacionada con las enfermedades del sistema circulatorio, que correspondieron el 23,4\% (11.940 muertes) de las defunciones de la región (MinSalud y Protección Social et al. 2013).

El envejecimiento de la población, la relación de la comorbilidad con la edad y de ambas con la mortalidad, hacen prever que las dimensiones de este problema irán en aumento. Todo ello, conlleva a un incremento en la incidencia y la prevalencia de enfermedades de carácter crónico, un mayor uso de la asistencia sanitaria y una mayor frecuencia de hospitalización (Villarejo \& Pancorbo, 2011). Esto representa un desafío para los sistemas de salud, donde las acciones son dirigidas a la reducción de la morbilidad y del alto costo que estas acarrean, convirtiéndose en una prioridad absoluta y una condición necesaria para el desarrollo sostenible de las Naciones.

La Fundación Hospital San Carlos es la primera institución de carácter privado en el país, cumpliendo 68 años de labor; actualmente, se especializa en la atención de nivel I, II, III y IV y cuenta con los servicios de consulta externa, urgencias, hospitalización, cirugía, cuidados intensivos en diferentes especialidades, entre otros. El Hospital dispone de 333 camas, 204 dedicadas al servicio de Medicina Interna, de las cuales, 12 son de aislamiento y 33 para el servicio de Geriatría, manejadas exclusivamente por geriatras (FHSC, 2016), para atender a una población de más de 5.429.827 adultos residentes en la cuidad de Bogotá (Secretaría Distrital, 2015). La FHSC cumple con variadas y complejas misiones, especialmente asistenciales, investigativas y docentes; tiene la visión de ser reconocidos, en el 2018, como el principal Centro de Alta Complejidad del Suroriente de Bogotá y un referente Nacional e Internacional en atención integral del adulto (FHSC, 2016).

Ante este panorama surge la pregunta de esta investigación: ¿Cuál es la comorbilidad asociada en el paciente geriátrico hospitalizado en la Fundación Hospital San Carlos? Los objetivos propuestos fueron: Analizar la comorbilidad en el paciente geriátrico de la FHSC hospitalizado en el 2014 y primer semestre del 2015. Determinar las principales comorbilidades de los pacientes que egresaron del servicio de geriatría e identificar las comorbilidades que generan mayor estancia hospitalaria.

\section{MATERIALES Y MÉTODOS}

Es un estudio cuantitativo de tipo analítico, retrospectivo. El método analítico es un camino para llegar a un resultado, mediante la descomposición de un fenómeno en sus elementos constitutivos (Lopera et al. 2010) y según la cronología de los hechos, se utilizó un diseño retrospectivo, donde el investigador observa la manifestación de algún fenómeno (v. dependiente) e intenta identificar, retrospectivamente, sus antecedentes o causas (v. independiente) (Cabrero et al. 2015). Se revisaron 2.599 historias clínicas de pacientes, con edades entre 65 y 100 años, quienes egresaron del servicio de geriatría en la Fundación Hospital San Carlos, en el 2014 y primer semestre de 2015.

La metodología para determinar la muestra: Se realizó a partir de muestreo aleatorio simple y la fórmula:

$$
\begin{aligned}
& n=\frac{k^{2} \times N \times p \times(1-p)}{e^{2} \times(N-1)+k^{2} \times p \times(1-p)}= \\
& =\frac{1,96^{2} \times 2599 \times 0,5 \times(1-0,5)}{0,1^{2} \times(2599-1)+1,96^{2} \times 0,5 \times(1-0,5)} \approx 93
\end{aligned}
$$

Dónde: $k$ corresponde a la constante asociada a la confiabilidad del $95 \%$; $N$ total de la población; $p$ probabilidad de éxito de encontrar comorbilidad; $1-p$ probabilidad de fracaso de encontrar comorbilidad; $e$ error de estimación considerado para este caso fue de $10 \%$. Se obtuvieron, de acuerdo a la fórmula, 93 historias clínicas, a partir de una muestra aleatorizada y que cumplían con los siguientes criterios de inclusión para el estudio: Historias clínicas de pacientes mayores de 65 años de edad, que fueron hospitalizados en el 2014 o 2015-I, servicio de egreso Geriatría y la unidad de análisis fueron las historias clínicas de los pacientes hospitalizados durante el 2014-2015-I.

La recolección de la información, se realizó a través de la revisión de las historias clínicas documentadas en el sistema 
del hospital HOSVITAL; para acceder a esta información, se obtuvo el aval del Comité de Ética Institucional. Se tuvieron en cuenta los datos de población durante el periodo requerido para, posteriormente, determinar la muestra, objeto de estudio y un registro de pacientes.

La información se consolidó en una plantilla en Excel. El instrumento de captura de las historias clínicas consta de cuatro componentes: datos de identificación del paciente, ingreso a la institución, servicios durante la hospitalización y egreso. La información se organizó en una base de datos, para su posterior análisis.

Para el análisis de la información, se utilizó la técnica de análisis de varianza y se determinó el número de veces del diagnóstico, durante la estancia hospitalaria y el grupo de diagnósticos que reportaban mayor prevalencia durante la estancia del paciente y se realizó a través del lenguaje de programación R Versión R-3.3.1.tar.gz (R Core Team, 2015).

Finalmente, la prevalencia de la información obtenida permitió generar una lista final de diagnósticos de comorbilidad en las historias revisadas.

Implicaciones éticas: Se tuvieron en cuenta todas las consideraciones éticas generales subrayados en la Declaración de Helsinki de 1960, acordados por la Asociación Médica Mundial, revisados en octubre del 2000 y promulgados en Colombia, mediante la Resolución No 008430 del 4 de octubre de 1993 del Ministerio de Salud de la República de Colombia, por la cual, se establecen las normas científicas, técnicas y administrativas para la investigación en salud. Estudio sin riesgo, se revisaron las historias clínicas y se asignaron códigos, para guardar la confidencialidad de la información.

\section{RESULTADOS Y DISCUSIÓN}

De las historias clínicas revisadas, 51,6\% correspondieron a población femenina y un $48,4 \%$, masculina; se encontraron en un rango de edad de 65 a 100 años, con un promedio de 80 años \pm 2 años.

De acuerdo a la revisión de las historias clínicas, se analizó la estancia hospitalaria y se determinó el número de veces que los diagnósticos se presentaron durante la misma; se encontraron 151 diagnósticos. La gráfica 1 presenta los once primeros diagnósticos, que corresponden a aquellos que cumplieron con una prevalencia mínima del $2 \%$.

Los principales diagnósticos que presentaron los sujetos de estudio, durante la estancia hospitalaria, corroboran la presencia de tres de los cuatro grupos de enfermedades crónicas no trasmisibles. Estas fueron: los problemas cardiovasculares (HTA, FC y CRPT); enfermedades respiratorias (EPOC) y Diabetes Mellitus (tipo II) y, las demás, generan una prevalencia total mayor a un $40 \%$ del total de los diagnósticos de egreso. Diferentes enfermedades correlacionadas por las características de los pacientes de edad avanzada.

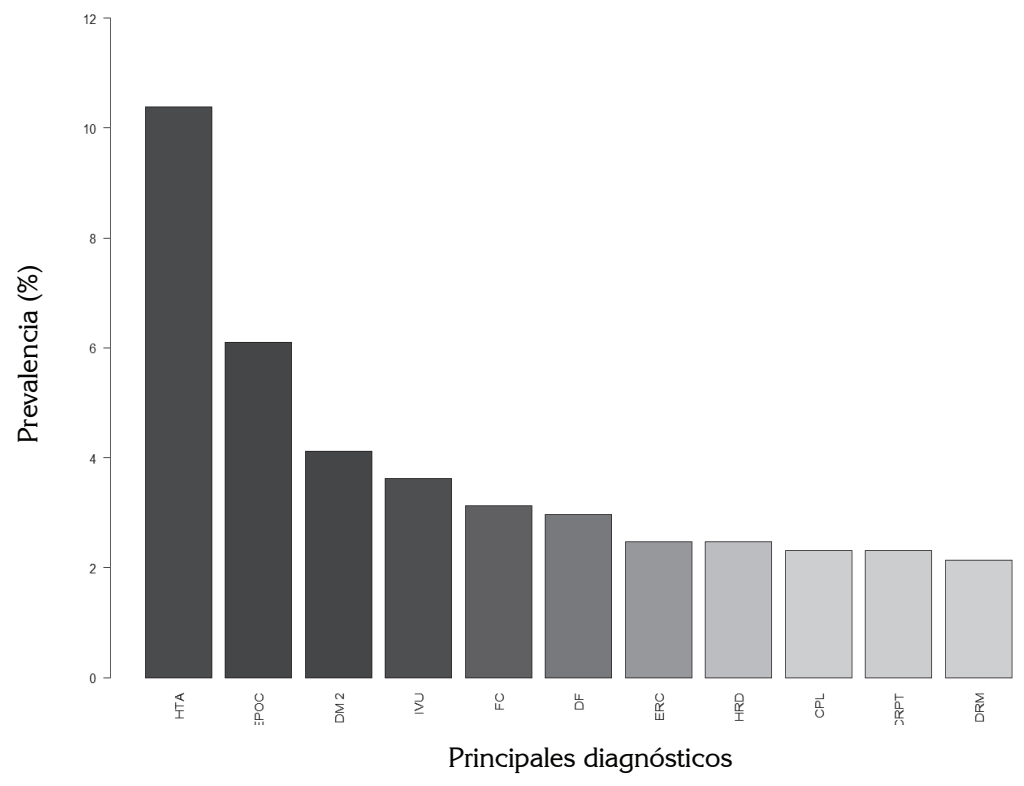

HTA: Hipertensión Arterial; EPOC: Enfermedad Pulmonar Obstructiva Crónica. DM 2: Diabetes Mellitus Tipo II; IVU: Infección de Vías Urinarias; FC: Falla Cardiaca; DF: Dependencia Funcional; ERC Enfermedad Renal Crónica; HRD: Hipotiroidismo; CPL: Cor Pulmonar; CRPT: Cardiopatía; DRM: Delirum.

Gráfica 1. Diagnósticos de mayor prevalencia. 
Estos resultados concuerdan con los presentados por Rojas, en un estudio realizado en Perú, con adultos mayores, con rangos de edad similares y patologías infecciosas, neoplásicas y crónicas no transmisibles (Rojas, 2010).

Igual que en el estudio realizado en un Hospital General en Galicia, España, en el que la totalidad de ingresos hospitalarios de los servicios de adultos, entre 2000 y 2009, se identificaron las 25 patologías crónicas más frecuentes: Cardiopatía Isquémica, Insuficiencia Cardíaca, Demencia, Enfermedad Pulmonar Obstructiva Crónica y Cáncer de cualquier localización y determinando las diferentes combinaciones de multimorbilidad (Matesanz et al. 2012).

El promedio de la estancia hospitalaria de los pacientes que egresaron del servicio de geriatría de la FHSC fue de $11 \pm 2$ días. \pm Promedio estándar de error. Coincide con lo encontrado en un estudio realizado en un Hospital de Lima, en el que se reportó una estancia hospitalaria de 13,6 días, en el grupo de personas mayores de 60 años (Tello et al. 2011), así como en un estudio efectuado en tres hospitales de Venezuela, con promedio de 10 días (Vargas \& Hernández, 2007).

Para el presente estudio, se determinó la prevalencia, a través del análisis de varianza, teniendo en cuenta los diagnósticos, cuyo promedio en días significó más del $40 \%$ de la estancia hospitalaria, con un resultado de 32 diagnósticos, que provocaron la prolongación de la misma.

Se reportaron, en número de días, los siguientes: Neumonía Asociada al Cuidado de la Salud, Enfermedad Renal Crónica, 22; Fístula Recto Vaginal, Hipoalbuminemia, Obstrucción Urinaria, 21; Discopatía, Distonía Aguda, 16; Hematoma, 15,2; Edema Pulmonar, 15; Lesión Renal Aguda, 14,3; Insuficiencia Cardiaca Congestiva, 14; Gota, Trauma, 13,5; Hidronefrosis, Temblor Esencial, 13; Bacteriemia, 12,5; Hernia, Síndrome Hemolítico, 12; Celulitis, 11,1; Cistotomía, Disfunción Multiorgánica, Neuralgia del Trigémino, 11; Infarto Agudo de Miocardio, 10,7; Secuelas de Accidente Cerebro Vascular, 10,6; Hemorragia de Vías Digestivas, Trombosis Venosa Profunda, 10,3; Aneurisma, Craneotomía, Enfermedad Arterial, Pancreatitis, Parkinson, Paraparesia Espástica, 10.

Las Neumonías Asociadas al Cuidado de la Salud presentaron una prevalencia en la prolongación de la estancia hospitalaria, de 22 días, lo que puede generar el aumento de los costos de hospitalización y de tratamiento que, en general, deberían durar de 7 a 10 días (Munive et al. 2013).

Como segundo diagnóstico importante, se evidenció la Enfermedad Renal Crónica ERC. Aunque los diferentes fenómenos que se relacionan con la aparición de daño renal, no son motivo de estudio en esta investigación y no se puede determinar la correlación; se evidencia que hay presencia diagnóstica de estos fenómenos en los datos obtenidos.

El Registro Renal Permanente Kaiser (Sistema Integral de Seguro Médico en California, EE.UU.) reporta que los pacientes con filtrado glomerular más reducido, mostraron una mayor tasa de hospitalización, mayores complicaciones cardiovasculares (cardiopatía coronaria, insuficiencia cardíaca, ictus isquémico y arteriopatía periférica) y mayor mortalidad (Górriz \& Otero, 2008).

Según la Sociedad Española de Nefrología, la ERC es una enfermedad predominante en la actualidad y afecta a un porcentaje importante de la población. Se relaciona con fenómenos o enfermedades de alta prevalencia, como el envejecimiento, la hipertensión arterial, la diabetes o la enfermedad cardiovascular. La ERC forma parte, frecuentemente, del contexto de comorbilidad que padecen enfermos seguidos por múltiples especialidades médicas, particularmente, por Atención Primaria, Medicina Interna, Cardiología, Geriatría, Endocrinología y cualquier otra especialidad médica o quirúrgica, que trate pacientes en riesgo de desarrollar ERC, sobre todo aquellos de edad avanzada (Gorostidi et al. 2014).

Los datos encontrados en el presente estudio indican que la comorbilidad encontrada en pacientes geriátricos hospitalizados en la Fundación Hospital San Carlos corresponde a aquellas enfermedades agudas y crónicas, de aparición repentina durante la estancia hospitalaria y que no necesariamente se vinculan con los estados de salud iniciales. La comorbilidad observada en la hospitalización de esta población y la identificación de los diagnósticos que prolongan la estancia hospitalaria, generan diferentes retos en la atención en salud.

Los pacientes que son atendidos en la FHSC tienen un alto grado de vulnerabilidad, por el grupo de edad al que representan y son los que más demandan atención en salud, como consecuencia de la comorbilidad y los cambios físicos, psicológicos y de funcionalidad inherentes al proceso de envejecimiento (Villareal \& Month, 2012).

De la presente investigación, se concluye que los principales diagnósticos asociados a la comorbilidad de los pacientes que egresaron del servicio de geriatría fueron 151 diagnósticos, de los cuales, prevalecen once, con más del $40 \%$.

Se identificó la presencia de 32 entidades como comorbilidades, que generan mayor estancia hospitalaria. Los diagnósticos de mayor prevalencia fueron enfermedades crónicas, con la ERC y agudas, como la NACS.

Conflicto de intereses: El manuscrito fue preparado y revisado con la participación de todos los autores, quienes de- 
claramos que no existe conflicto de intereses que ponga en riesgo la validez de los resultados presentados.

\section{BIBLIOGRAFÍA}

1. ALONSO, P.; BERNABEU, M.; CASARIEGO, E.; RICO, M.; ROTAECHE, R.; SÁNCEZ, S. 2013. Desarrollo de guías de práctica clínica en pacientes con comorbilidad y pluripatología. Sociedades Científicas de España. 1-34. Disponible desde Internet en: http:// www.semfyc.es/pfw_files/cma/biblioteca/_DesarrolloGuiasPluripatologia.pdf (con acceso 10/02/2016).

2. ARANGO, V.; PÉREZ, M.; GUTIÉRREZ, L. 2012. Enfermedades crónicas y síndromes geriátricos: impacto en la capacidad funcional. Foro envejecimiento y salud. 1-4. Disponible desde Internet en: http://www.geriatria.salud.gob.mx/descargas/foro/FS_ENFERMEDADES_CRONICAS.pdf (con acceso 08/03/2016).

3. BERNABEU, M.; ALONSO, P.; RICO-BLÁZQUEZ, M.; ROTAECHE, R.; SÁNCHEZ, S.; CASARIEGO, E. 2014. Desarrollo de guías de práctica clínica en pacientes con comorbilidad y pluripatología. Atención Primaria (España). 46(7):1-8.

4. BOULT, C.; WIELAND, G. 2010. Comprenhensive primary care for older patients with multiple chronic conditions. JAMA. 304(17):1936-1943.

5. CABRERO, J.; MARTÍNEZ, M.; MARTÍNEZ, N. 2015. Diseño de la investigación. Disponible desde Internet en: http://www.aniorte-nic.net/apunt_metod_investigac4_4.htm (con acceso el 01/03/2016).

6. FEINSTEIN, A.R. 1970.The pre-therapeutic classification of co-morbidity in chronic diseases. J. Chronic Diseases. 23:455-469.

7. FERNÁNDEZ, J.; BUSTOS-VÁZQUEZ, E. 2016. Multimorbilidad: bases conceptuales, modelos epidemiológicos y retos de medición. Biomédica. 33(4):1-52.

8. FUNDACIÓN HOSPITAL SAN CARLOS -FHSC-. 2016. Fundación Hospital San Carlos. Disponible desde Internet en: http://fhsc.org.co/ (con acceso el 12/02/2016).

9. GÓMEZ, R.; MARTÍNEZ, M.; FORMIGA, F.; ALEMÁN, J.; CAMAFORT, M.; GALVE, E.; LOBOS, J. 2014. Tratamiento de los factores de riesgo vascular en el paciente mayor de 80 años. Medicina Clínica (España). 143(3):133-145.
10. GOROSTIDI, M.; SANTAMARÍA, R.; ALCÁZAR, R.; FERNÁNDEZ-FRESNEDO, G.; GALCERÁN, J.; GOICOECHEA, M.; RUILOPE, L. 2014. Documento de la sociedad española de nefrología sobre las guías KDIGO para la evaluación y el tratamiento de la enfermedad renal crónica. Nefrología (España). 34(3):302-316.

11. GÓRRIZ, J.; OTERO, A. 2008. Impacto socio sanitario de la enfermedad renal crónica avanzada. Nefrología (España). 28(3):7-15.

12. KARLAMANGLA, A.; TINETTI, M.; GURALNIK, J.; STUDENSKI, S.; WETLE, T.; REUBEN, D. 2007. Comorbidity in older adults: Nosology of impairment, diseases, and conditions. J. Gerontol. A Biol. Sci. Med. Sci. $62^{\mathrm{a}}: 296-300$.

13. LAPORTE, A. 2012. La valoración de la multimorbilidad en personas de edad avanzada. Un área importante de la valoración geriátrica integral. Rev. Española Geriatría y Gerontología. 47(2):47-48.

14. LOPERA, J.; RAMÍREZ, C.; ZULUAGA, M.; ORTIZ, J. 2010. El método analítico cómo método natural. Nómadas. Rev. Crítica de Ciencias Sociales y Jurídicas (Colombia). 25(1):327-353.

15. MARTÍNEZ, N.; GAMINDE, I. 2011. Índices de comorbilidad y multimorbilidad en el paciente anciano. Medicina Clínica. 136(10):441-446.

16. MATESANZ, M.; ÍINIGUEZ, I.; RUBAL, D.; PÉRTEGA, S.; CONDE, J.; CASARIEGO, E. 2012. Multimorbilidad en los servicios médicos hospitalarios : un problema clínico y de gestión. Medicina Interna (España). 73:15-19.

17. MINISTERIO DE SALUD Y PROTECCION SOCIAL MINSALUD-; DEMOGRAFÍA, DIRECCIÓN DE EPIDEMIOLOGÍA; ASIS, GRUPO. 2013. Análisis de situación de salud según regiones Colombia. 1-160. Disponible desde Internet en: https://www. minsalud.gov.co/Documentos\%20y\%20Publicaciones/An\%C3\%A1lisis\%20de\%20situaci\%C3\%B3n\%20 de\%20salud\%20por\%20regiones.pdf [con acceso el 29/02/2016].

18. MINISTERIO DE SALUD Y PROTECCIÓN -MINSALUD-. 2012. Indicadores básicos 2012. Situación de salud en Colombia. Ministerio de salud, (32), 65. Disponible desde Internet en: https://www.minsalud.gov. $\mathrm{co} /$ sites/rid/Lists/BibliotecaDigital/RIDE/VS/ED/PSP/ Indicadores-basicos-en-salud-2012.pdf (con acceso el 15/02/2016). 
19. MUNIVE, A.; ORTIZ, G.; DUEÑAS, C. 2013. Consenso colombiano de neumonía nosocomial. Infectio (España). 17(1):6-18.

20. ORGANIZACIÓN MUINDIAL DE LA SALUD -OMS-. 2014. Informe sobre la situación mundial de las enfermedades no transmisibles, 1-18. Disponible desde Internet en: http://doi.org/ISBN: 978924156422 9 (con acceso el 19/02/2016).

21. ORGANIZACIÓN MUNDIAL DE LA SALUD -OMS-. 2015. Informe Mundial sobre el Envejecimiento y la Salud. 2015. Disponible desde Internet en: http:// www.who.int/ageing/publications/world-report-2015/ es/ (con acceso el 16/02/2016).

22. ORGANIZACIÓN MUINDIAL DE LA SALUD -OMS-. 2016. Envejecimiento y ciclo de vida. Disponible desde Internet en: http://www.who.int/features/factfiles/ ageing/ageing_facts/es/ (con acceso el 08/02/2016).

23. PAN AMERICAN HEALTH ORGANIZATION -PAHO-. 2015. Las enfermedades no transmisibles nuestro reto. Disponible desde Internet en: http://www.paho.org/col/index.php?option=com content\&view $=$ article\&id $=1756$ :las-enfermedadesno-transmisibles-ent-nuestro-reto \&Itemid $=487$ (con acceso el 09/03/2016).

24. R CORE TEAM. 2015. R: A language and environment for statistical computing. R Foundation for Statistical Computing. Vienna, Austria. Disponible desde Internet en: https://www.r-project.org/ (con acceso el 11/02/2016).

25. ROBLEDO, R.; ESCOBAR, F. 2010. Las enfermedades crónicas no transmisibles en Colombia. Bol. Observ. Salud (Colombia). 3(4):1-9.

26. ROJAS, D. 2010. Morbilidad y mortalidad del adulto mayor en un servicio de medicina de un hospital general del Perú. Rev. Peruana de Epidemiología. 14(2):99-107.

27. ROSAS, O.; GONZÁLEZ, E.; BRITO, A.; VÁZQUEZ, O.; PESCHARD, E.; GUTIÉRREZ, L. M.; GARCÍA,
E. 2011. Evaluación de la comorbilidad en el adulto mayor. Rev. Med. Inst Mex Seguro Soc (México). 49(2):153-162. Disponible desde Internet en: http:// www.medigraphic.com/pdfs/imss/im-2011/im112j. pdf (con acceso el 02/03/2016).

28. SALECH, F.; JARA, R.; MICHEA, L. 2012. Cambios fisiológicos asociados al envejecimiento. Rev. Méd. Clínica (Colombia). 23(1):19-29.

29. SECRETARÍA DISTRITAL DE PLANEACIÓN. 2015. Aspectos Demográficos. Disponible desde Internet en: http://www.sdp.gov.co/PortalSDP/InformacionTomaDecisiones/Estadisticas/ProyeccionPoblacion (con acceso el 05/03/2016).

30. TELLO, T.; VARELA, L.; ORTIZ, J.; CHAVEZ, H. 2011. Estancia hospitalaria y mortalidad en adultos mayores hospitalizados en un hospital general de Lima Metropolitana, 1997-2008. Rev. Med. Hered (Perú). 22(1):23-28.

31. VALDERAS, M.; STARFIELD, B.; SIBBALD, B.; SALISBURY, C.; ROLAND, M. 2009. Defining comorbidity: Implications for understanding health and health services. Ann. Fam. Med. 7:357-363.

32. VARGAS, V.; HERNÁNDEZ, E. 2007. Indicadores de gestión hospitalaria. Rev. Inst. Nal. Enf. Resp. Mex. (México). 13(3):444-454.

33. VILLAREAL, G.; MONTH, A. 2012. Condición socio familiar, asistencial y de funcionalidad del adulto mayor de 65 años en dos comunas de Sincelejo (Colombia). Salud Uninorte (Colombia). 28(1):75-87.

34. VILLAREJO, L.; PANCORBO, P. 2011. Diagnósticos de enfermería, resultados e intervenciones identificadas en pacientes ancianos pluripatológicos tras el alta hospitalaria. Gerokomos (España). 22(4):152-161.

Recibido: Abril 16 de 2016

Aceptado: Agosto 17 de 2016

Cómo citar

Contreras-Acevedo, P.J.; Vargas, L.D.; Sanjuanelo Corredor, D.W.; Robayo, J.P.; Niño Cardozo, C.L. 2016. Comorbilidad del paciente hospitalizado en el servicio de geriatría en la fundación hospital San Carlos. Rev. U.D.C.A Act. \& Div. Cient. 19(2): 267-273. 